\title{
Cardiac Autonomic Neuropathy- An ECG Correlation in Type 2 Diabetes
}

\author{
Subashini Vijayan ${ }^{1}$, Ganesamoorthy K. ${ }^{2}$, Usha Padmini ${ }^{3}$, Ravikumar T. ${ }^{4}$ \\ ${ }^{1}$ Assistant Professor, Department of Medicine, Government Medical College and ESI Hospital, Coimbatore, Tamilnadu, \\ India. ${ }^{2}$ Associate Professor, Department of Medicine, Government Medical College and ESI Hospital, Coimbatore, \\ Tamilnadu, India. ${ }^{3}$ Associate Professor, Department of Medicine, Government Medical College and ESI Hospital, \\ Coimbatore, Tamilnadu, India. ${ }^{4}$ Professor and HOD, Department of Medicine, Government Medical College and ESI \\ Hospital, Coimbatore, Tamilnadu, India.
}

\section{ABSTRACT}

\section{BACKGROUND}

Cardiac autonomic neuropathy is a serious complication with poor prognosis in type 2 diabetes. It is asymptomatic in diabetes. It results in increased incidence of silent myocardial infarction and ischemia and left ventricular dysfunction. Diabetic cardiac autonomic neuropathy (CAN) a serious microvascular complication with poor prognosis. Diabetic cardiac autonomic neuropathy (CAN), a major complication seen in one-sixth of insulin dependent type 1 diabetes and one-third of non-insulin dependent diabetic patients, is related with increased morbidity. We wanted to study the ECG and ECHO changes in type 2 diabetes to diagnose cardiac autonomic neuropathy.

\section{METHODS}

This was a case control study and sample size was taken for convenience. Patients attending diabetes and medical OPD in a teaching hospital were selected according to inclusion and exclusion criteria. Hundred patients of type 2 diabetes mellitus of more than five years duration and 100 age and sex matched controls without any history of diabetes were selected. ECG and ECHO were done in all cases and controls.

\section{RESULTS}

A total of 100 type 2 diabetes patients and 100 age matched controls were studied. QTc interval prolongation occurs with development of CAN. Prolongation of QTC interval is well correlated with Cardiac Autonomic Neuropathy in type 2 diabetes.

\section{CONCLUSIONS}

QTc interval is easily measurable and is a non-invasive method. It is the earliest sign of cardiac autonomic neuropathy. It is prolonged in type 2 diabetes patients with severe cardiac autonomic dysfunction. They have a high risk of developing silent myocardial infarction and sudden death. Early diagnosis and early control of sugar will prevent morbidity in diabetic individuals.

\section{KEY WORDS}

Cardiac Autonomic Neuropathy, Type 2 Diabetes, Myocardial Infarction, Coronary Artery Disease QT Dispersion ECG

\author{
Corresponding Author: \\ Subashini Vijayan, \\ \#202, Third Floor q2 Apartment, \\ ESI Quarters, Varadharajapuram, \\ Coimbatore-641015, Tamilnadu, India. \\ E-mail: sssubavijayan@gmail.com
}

DOI: $10.14260 /$ jemds/2019/593

Financial or Other Competing Interests: None.

How to Cite This Article:

Vijayan S, Ganesamoorthy K, Padmini $U$, et al. Cardiac autonomic neuropathy-an ECG correlation in type 2 diabetes. J. Evolution Med. Dent. Sci. 2019;8(35):2733-2737, DOI: $10.14260 /$ jemds/2019/593

Submission 26-03-2019,

Peer Review 18-08-2019,

Acceptance 24-08-2019,

Published 02-09-2019.

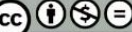




\section{BACKGROUND}

Worldwide, incidence of Type 2 diabetes is rising very rapidly. It was estimated that about 140 million people are diabetics in the year of $1996^{6}$. This estimates doubles in another 30 years mainly in developing and underdeveloped countries more prevalent in Asian and African- Caribbean and in some inner urban areas. Asian people have higher incidence of diabetic nephropathy (DN) and coronary artery disease (CAD). Diabetes affect almost every organ in the body. It is divided into micro and macro vascular complications. "Diabetes is easy to diagnose, but can be managed with negligent ease by those inclined to do so" RB Tattersall, 19901. Diabetic cardiac autonomic neuropathy (CAN) a serious microvascular complication with poor prognosis. Diabetic cardiac autonomic neuropathy (CAN), major complication seen in one-sixth of insulin dependent type 1 diabetes and one-third of non-insulin dependent diabetic patients, related with increased morbidity ${ }^{2}$. The CAN Subcommittee of Toronto Consensus Panel on Diabetic Neuropathy defines CAN as an "impairment of cardiovascular autonomic control in patients with established diabetes after excluding other causes ${ }^{3}$."

It results in postural hypotension, exercise intolerance, enhanced intra operative instability, an increased incidence of silent myocardial infarction and ischemia and left ventricular (LV) dysfunction ${ }^{4}$. CAN increases with obesity, age, poor glycaemic control, and may be with duration of diabetes. Diabetes was recently demonstrated as the strongest predictor of atrial fibrillation progression and patients with diabetes and CAN frequently have asymptomatic episodes of atrial fibrillation with silent arrhythmia progression. ${ }^{5}$ The association between CAN and QT interval prolongation demonstrated in more studies and it predispose to sudden cardiac death in diabetes. Increased QT dispersion suggested as a marker of diabetic autonomic neuropathy. Sacre et al. evaluated the association of CAN with LV dysfunction. Association between diastolic function and CAN, independent of metabolic factors and other contributors to $\mathrm{LV}$ dysfunction ${ }^{6}$ The relative predominance of sympathetic activity at the onset of CAN would stimulate the reninangiotensin aldosterone system, which not only increases the hemodynamic stresses and energetic requirements of the left ventricle by sodium retention and peripheral vasoconstriction but may also exert direct noxious effects on cardiomyocytes (Apoptosis and regression to a fetal phenotype) and changes in the nature of the extracellular matrix. (Stimulation of myocardial fibrosis), which may further alter the architecture and impair the performance of the left ventricle. ${ }^{7}$

Such sympathetic hyperactivity, in combination with regional myocardial denervation present in a more advanced stage of CAN, has been recently shown to lead to diminished coronary blood flow reserve and diastolic dysfunction in diabetic patients with early microangiopathy ${ }^{8}$. The confirmation of the presence of CAN in otherwise healthy type 2 diabetes patients, and its independent association with resting diastolic dysfunction, is important. Several treatment interventions including graded exercise, cardioactive drugs, and intensification of treatment for the multiple risk factors for CAN that are shared with those for macrovascular disease have been shown to improve the functional deficiency in the autonomic nervous system. ${ }^{7}$ The ECG offers a quick, noninvasive clinical and research screen for the early detection of cardiovascular disease in diabetes. QTc interval $>460 \mathrm{~ms}$ has been associated with a 2-fold increased cardiovascular and all-cause mortality risk in a 5 -year prospective population study in individuals with type 2 diabetes. Furthermore, individuals with baseline CAN were 2 times more likely to die compared with individuals without CAN.In a recent case control study of 682 people with coronary heart disease and diabetes but no history of sudden cardiac death, prolongation of QTc interval was a significant predictor of sudden cardiac death even among individuals with a normal or borderline QTc interval. However, idiopathic abnormal QTc interval prolongation was associated with a higher (5-fold) increased risk of sudden cardiac death Therefore, non-invasive identification of early stages of CAN using QT dispersion and 2D echo may emphasize the need for intensive control of cardiovascular risk factors, thereby reducing the risk of premature mortality.

The most widely used tests assessing cardiac parasympathetic function are based on the time-domain heart rate response to deep breathing, the Valsalva manoeuvre, and postural change. Of these tests, heart rate response to deep breathing has the greatest specificity9. Cardiovascular sympathetic function is assessed by measuring the blood pressure response to orthostatic change and the Valsalva maneuver ${ }^{10}$. The performance of these tests should be standardized, and the influence of confounding variables such as medications, hydration, and antecedent activity should be minimized. Age normative values should be used. As already mentioned, the combination of cardiovascular autonomic tests with sudomotor function tests may allow a more accurate diagnosis of CAN. Bedside tests for CVS are used to exclude autonomic neuropathy. Cardiac autonomic function tests are done using cans analyser. Doing so not only helps reduce the risk of heart disease, but also lowers the risk of diabetic eye, kidney and nerve disease, each of which patients want to avoid. Diabetic autonomic neuropathy has been called a "silent killer," because so few patients realize that they suffer from it, and yet its effects can be so lethal. With a brief, 15-minute test that we can administer in the office, and some relatively modest interventions, we can help many patients live longer, healthier lives.

Treatment for Cardiac Autonomic Neuropathy is available. The best chance for intervention and changes of prognostics for a CAN patient is the early diagnosis ${ }^{10}$. The treatment is based on non-pharmacological treatments that include weight loss and improvements to insulin resistance, since both are associated with sympathetic hyperactivity, and exercises must be of low to moderate intensity ${ }^{7}$ Pharmacological measures, such as ACE inhibitors and betablockers, can contribute to the attenuation of CAN symptoms. ${ }^{11}$ Therapies whose focus is, exclusively, the action of the sympathetic nervous system still require further studies, such as (i) hyperstimulation of the vagus nerve; (ii) renal sympathetic ablation; (iii) resection of the carotid body; and (iv) electrical stimulation of the baroreceptor. Two classes of drugs for DM2 treatment, iSGLT2, and aGLP-1, have shown a reduction in mortality due to cardiovascular disease using mechanisms not often linked to coronary artery disease. In a non-pre-specified analysis of the Empa-REG, ${ }^{12}$ it 
was suggested that: among patients included in the study who suffered a fatal cardiovascular event, 137 (5.3\%) were in the placebo group, while $90(3.8 \%)$ were being treated with $10 \mathrm{mg} / \mathrm{d}$ empagliflozin and 82 (3.5\%) with $25 \mathrm{mg} / \mathrm{d}$ empagliflozin. Sudden death was the cause of death for 38 patients in the placebo group, 30 in the $10 \mathrm{mg} / \mathrm{d}$ empagliflozin group, and 23 in the $25 \mathrm{mg} / \mathrm{d}$ empagliflozin group. Thus, sudden death was the cause for $1.6 \%$ of all deaths in the placebo group, against $1.1 \%$ in the group treated with empagliflozin, which represented a reduction of $31.25 \%$ in deaths by sudden death in the empagliflozin group. Studies using animals have demonstrated the action of the iSGLT2 in reducing the sympathetic hyperactivity ${ }^{13}$.

In another sub analysis also not pre-specified in the Leaderstudy, 278 patients progressed to death in the placebo group, against 219 patients in the treated group. Of these, sudden death was the cause of death for $74(1.6 \%)$ of patients in the placebo group, against $51(1,1 \%)$ in the liraglutide group, representing a $31.25 \%$ reduction in deaths by sudden death. Thus, these two classes of drugs, iSGLT2 and aGLP1, are found to be possible attenuators of sudden death, perhaps due to their, at least partial, impact on the autonomic nervous system.

\section{METHODS}

Patients attending diabetes or medical op was selected according to inclusion and exclusion criteria. Data collected from all those who fulfilled the inclusion and exclusion criteria after taking a detailed case history and obtaining a written informed consent. They were subjected to detailed clinical examination. Baseline data including age and sex, detailed medical history including conventional risks factors, clinical examinations and relevant investigations are included as part of the methodology. For all the subjects standing height and weight were measured. Hundred patients of type 2 diabetes mellitus of more than five years duration and 100 age and sex matched controls without any history of diabetes was selected. Inclusion criteria include diabetes mellitus diagnosed according to World Health Organization (WHO) criteria and duration more than or equal to five years duration. Those with Ischaemic Heart Disease/structural heart diseases, patients on pacemakers, chronic obstructive pulmonary disease, secondary diabetes mellitus, antihypertensive and anti-arrhythmic drugs dyselectrolytemia hyperthyroidism or hypothyroidism, type 1 diabetes gestational diabetes were excluded.

Cardiac autonomic function tests were be done in all cases. Interpretation of the test was based on the works of Ewing and Clarke. For grading of cardiovascular autonomic function, results are classified into normal, mild and severe (Scores 0, 1, 2 respectively). An overall score of ' 0 ' or ' 1 ' was considered normal, score 2, 3, 4 were considered mild and score $\geq 5$ were judged was severe autonomic function. Patients were divided into three groups Group 1 Severe autonomic neuropathy. Group 2 Early autonomic neuropathy Group 3 No autonomic neuropathy. A 12 lead ECG was taken in all patients at $50 \mathrm{~mm} / \mathrm{second}$. QT interval was taken from the onset of QRS complex to the end of T wave. QT was then corrected for heart rate using the Bazette's formulae QTc dispersion were calculated from ECG. All patients were undergone echocardiography. Patients with diastolic dysfunction were subject ted to cardiac stress testing to exclude ischemic disease. QTCc dispersion of subjects were compared among patients with and without autonomic neuropathy. All the data will be entered in proforma (Enclosed).The statistical software using SPSS version 20.0 was used for the analysis of data and MS word and Excel have been used to generate graphs, tables etc. Relation between age sex and autonomic neuropathy were assessed by chi square test.

\section{RESULTS}

This study consists of 100 type 2 diabetic patients and 100 age and sex matched healthy controls. Among 100 patients $9 \%$ between $31-40$ years of age $43 \%$ between $41-50$ years $42 \%$ between $51-60$ years $6 \%$ were between $61-70$ years of age.

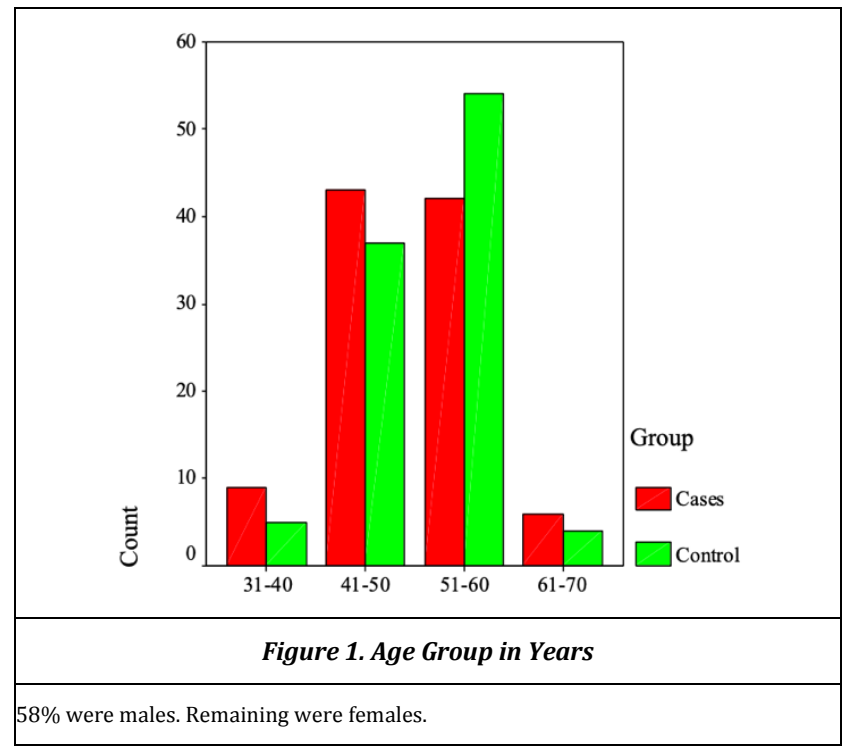

ECG, CAN Function assessed using analyser, ECHO were done for them. The prevalence of can in study group is high. This study showed significant relation between QTD, CAN grades and LVDD.

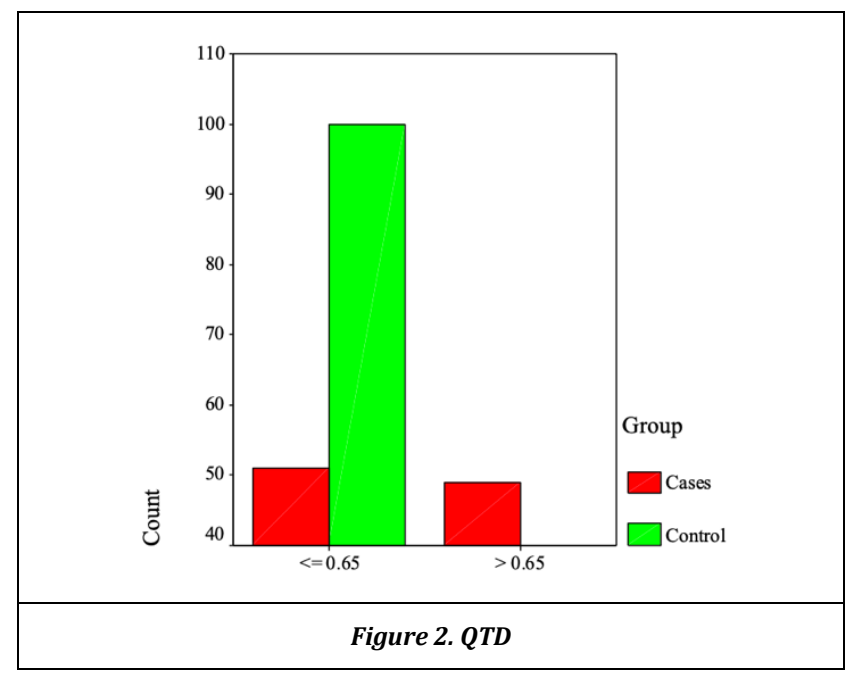


Cardiac autonomic neuropathy more common with longer duration of diabetes. It is associated with obesity and glycaemic control. Its incidence increases with age. More common among 50-60 years of age. Prevalence of diastolic dysfunction assessed by 2D echo is $46 \%$. It is relatively higher incidence in diabetics than healthy individuals. Its incidence also increases with age and duration of diabetes.

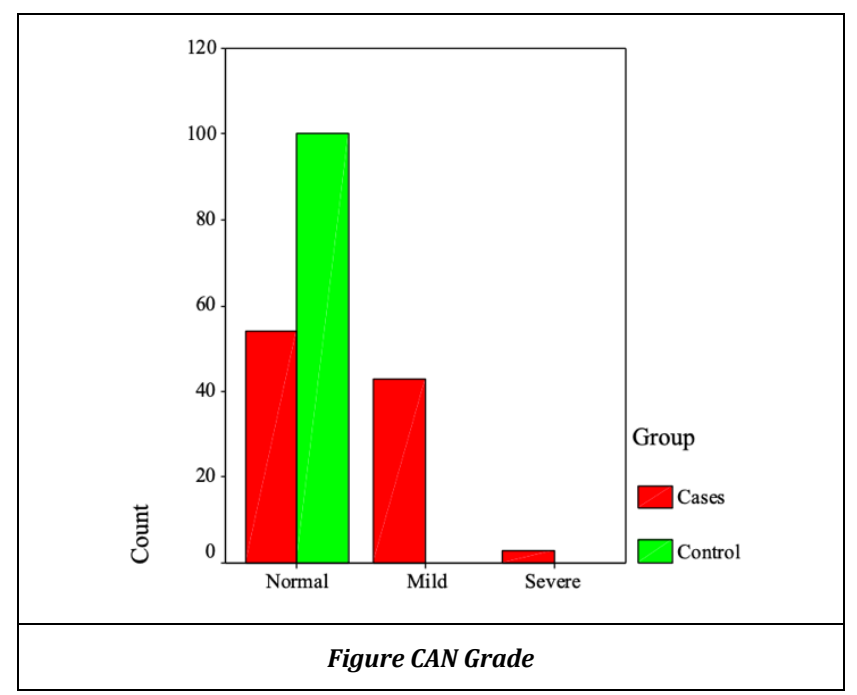

There is a significant association with prolongation of QT interval and cardiac autonomic neuropathy. There is association between cardiac autonomic neuropathy and diastolic dysfunction.

\section{DISCUSSION}

Risk of heart failure are two to three-fold larger in diabetes when compared to non-diabetes. In the early stages of the disease diastolic dysfunction is the only abnormality. 2D echo is used to assess diastolic dysfunction. QT dispersion that reflects spatial inhomogeneity in ventricular repolarization are associated with increased risk of certain arrhythmias and sudden cardiac death in type 2 diabetic patients and general population. Several studies, but not all, have found a significantly greater QT dispersion in diabetics Pappachan J M et al studied the utility of prolongation of corrected QT interval (QTc) in the ECG to diagnose CAN in patients with diabetes. They calculated the sensitivity and specificity of QTc prolongation for the diagnosis of CAN were $77 \%$ and $62.5 \%$ in type 1 and $76.5 \%$ and $75 \%$ in type 2 , respectively. They concluded that QTc interval in ECG can be used to diagnose CAN with reasonable sensitivity and specificity. This value of sensitivity and specificity correlates with our study. In another study by Jayaprasad et al found association between QTD and cardiac autonomic neuropathy in diabetes. In that study among 50 patients $42 \%$ had severe autonomic neuropathy and $24 \%$ had early autonomic neuropathy. Psallas et al conducted a study which showed QTD predict mortality in diabetes and it is associated with worse prognosis. Patients in our hospital showed high prevalence of cardiovascular risk factors such as obesity and poor glycaemic control. This situation offers a opportunity to study the effect of QTD as a proxy for electrophysiological phenomenon. Our study shows the deleterious effect of such risk factors on QTD particularly in long standing diabetes. In multivariate analysis QTD was predicted by sex, age, BMI and HbA1c. As expected, patients with diabetes had higher risk of developing QT prolongation than in non-diabetics. In a metaanalysis recently concluded that measurement of QTD is a more accurate test for autonomic failure in diabetes. Hyperglycaemia causes ventricular instability by increased sympathetic activation. In our study there is no correlation among sex smoking alcohol with QTD or CAN. In some other study conducted showed significant association of sex and QT prolongation. C P Mathur et al studied 50 patients with diabetes with 20 normal controls to understand the relationship to CAN with QTc interval. There were 15 (78.94\%) cases with QTc prolongation out of 19 diabetics with CAN. None of the diabetics without CAN or control subjects had QTc prolongation. It was observed to have sensitivity o9f $82.6 \%$ and specificity of $100 \%$. This value of sensitivity matches with our study, but it does not correlates with specificity value. There are certain limitations in this study. The study size is small, and hence necessitates the need of a larger study with wide range of study population. Lack of clinical follow up study. Mortality such as sudden cardiac death cannot be assessed so that we can confirm the influences of autonomic neuropathy Silent ischemia cannot be ruled out by coronary angiogram. More adverse clinical status of patients with CAN makes it difficult to isolate the direct effects of this complication on LV function, even after statistical adjustment. Congenital long QT syndrome cannot be ruled out.

\section{CONCLUSIONS}

CAN is a common microvascular complication in type $2 \mathrm{DM}$. Prevalence of cardiac autonomic neuropathy is high in long standing diabetics and rises with duration of diabetes. Its incidence is associated poor glycaemic control and obesity. QTC dispersion is significantly high in diabetics with autonomic neuropathy. ${ }^{4}$ QTc prolongation and QT dispersion are useful parameters in high risk groups and can be used to identify cardiac autonomic neuropathy. It is a simple noninvasive and cost-effective diagnostic tool. By using these tools, we can identify the subset of people who are at risk of sudden cardiac death.

\section{REFERENCES}

[1] Ziegler D. Diabetic cardiovascular autonomic neuropathy: prognosis, diagnosis and treatment. Diabetes Metab Rev 1994;10(4):339-83.

[2] Sivieri R, Veglio M, Chinaglia A, et al. Prevalence of QT prolongation in a type 1 diabetic population and its association with autonomic neuropathy. The Neuropathy Study Group of the Italian Society for the Study of Diabetes. Diabet Med 1993;10(10):920-4.

[3] Pfeifer MA, Weinberg CR, Cook DL, et al. Autonomic neural dysfunction in recently diagnosed diabetic subjects. Diabetes Care 1984;7(5):447-53. 
[4] Sacre JW, Franjic B, Jellis CL, et al. Association of cardiac autonomic neuropathy with subclinical myocardial Dysfunction in type 2 diabetes. J Am Coll Cardiol Img 2010;3(12):1207-15.

[5] Whitsel EA, Boyko EJ, Siscovick DS. Reassessing the role of QTc in the diagnosis of autonomic failure among patients with diabetes: a meta-analysis. Diabetes Care 2000;23(2):241-7.

[6] Veglio M, Borra M, Stevens LK, et al. The relation between QTc interval prolongation and diabetic complications: The EURODIAB IDDM Complications Study Group. Diabetologia 1999;42(1):68-75.

[7] Vinik AI, Maser RE, Mitchell BD, et al. Diabetic autonomic neuropathy. Diabetes Care 2003;26(5):1553-79.

[8] Ewing DJ, Martyn CN, Young RJ, et al. The value of cardiovascular autonomic function tests: 10 years of experience in diabetes. Diabetes Care 1985;8(5): 491-8.
[9] Kahn R. Proceedings of a consensus development conference on standardized measures in diabetic neuropathy. Autonomic nervous system testing. Diabetes Care 1992;15(8):1095-103.

[10] Takebayashi K, Aso Y, Sugita R, et al. Clinical usefulness of corrected QT intervals in diabetic autonomic neuropathy in patients with type 2 diabetes. Diabetes Metab 2002;28(2):127-32.

[11] Vinik AI, Ziegler D. Diabetic cardiovascular autonomic neuropathy. Circulation 2007;115(3):387-97.

[12] Pappachan JM, Varughese GI, Sriraman R, et al. Diabetic cardiomyopathy: pathophysiology, diagnostic evaluation and management. World J Diabetes 2013;4(5):177-89.

[13] Vinik AI, Erbas T. Recognizing and treating diabetic autonomic neuropathy. Cleve Clin J Med 2001;68(11):928-30, 932, 934-44. 